\title{
A Multipronged Intervention for Treatment of Psychotic Symptoms from Post-football Traumatic Brain Injury in an Adolescent Male: A Case Report
}

\author{
Dev Ashish, PhD ${ }^{1}$ Ariana Stickel, MA ${ }^{1} \quad$ Alfred Kaszniak, $\mathrm{PhD}^{1} \quad$ Catherine Shisslak, $\mathrm{PhD}^{1}$ \\ ${ }^{1}$ Department of Psychology, University of Arizona, Tucson, Arizona \\ Curr Res Concussion 2017;4:e32-e37. \\ Address for correspondence Catherine Shisslak, PhD, University of \\ Arizona, 1503 E University Boulevard, Tucson, AZ 85716, Arizona \\ (e-mail: cms@u.arizona.edu).
}

\begin{abstract}
Keywords

- adolescent TBI

- psychosis

- Acceptance and Commitment Therapy

- family therapy

Introduction Traumatic brain injury (TBI) is common in adolescents. TBI can result in impaired cognitive functioning and mood disturbance. In some cases, TBI results in psychotic symptoms. There is little documentation for treatment of psychotic symptoms resulting from TBI.

Case presentation The present case study reviews an adolescent male who was seen in an outpatient behavioral health clinic, following a football-related TBI. The TBI resulted in postconcussive syndrome including psychotic symptoms (i.e., visual and auditory hallucinations) and increased anxiety.

Management and Outcome The adolescent underwent 12 individual sessions of Acceptance and Commitment Therapy (ACT) and 7 sessions of family therapy for his anxiety and psychotic symptoms. He also underwent Vestibulo-Ocular Therapy. At the end of treatment, the patient's anxiety symptoms and those related to post-concussive syndrome were in remission with no hallucinations experienced for $>6$ months.

Discussion The results provide encouragement for systematic randomized controlled trials of individual and family behavioral interventions as part of an integrated treatment approach for mild TBI.
\end{abstract}

Traumatic brain injury (TBI) is common among children and adolescents $^{1,2}$ with a peak in mid-adolescence, ages 15 to 17 years. $^{3}$ According to Center for Disease Control and Prevention data, in 2009, an estimated 2,48,418 children and adolescents (age 19 or younger) were treated for concussion or TBI in emergency departments in the United States, and the diagnosis of concussion or TBI in this age group rose $57 \%$ between 2001 and 2009. ${ }^{4}$ Most adolescent TBIs are in males and are typically sports-related injuries. ${ }^{1,3,5}$ Individuals who have endured a TBI, even mild TBI, otherwise called concussion, often experience a wide-range of symptoms that negatively impact quality of life, including physical, cognitive, and psychological symptoms. ${ }^{6}$ Personality changes (e.g., increased aggression and disinhibition) are the most common psychological changes following a TBI in children and adolescents. ${ }^{7,8}$ Depression and anxiety can also develop following TBI. $^{9}$ In

received

November 28, 2016 accepted after revision August 4, 2017 some individuals, such symptoms resolve within weeks, while in others, these symptoms remain for years, and a history of multiple TBIs increases risk for developing chronic depression and cognitive impairments later in life. ${ }^{10}$

Hallucinations are a less common form of psychological illness following TBI. ${ }^{11-15}$ Estimates of psychotic symptoms (e.g., hallucinations and delusions) are calculated to be present in as many as 20 to $25 \%$ of all TBI cases, and the presence of psychotic symptoms does not seem to be related to severity of head injury. ${ }^{13}$ TBIs also increase the risk of developing chronic psychosis symptoms (e.g., schizophrenia ${ }^{16,17}$ ).

In a recent case, ${ }^{11}$ a male with a history of TBI in early adulthood reported experiencing ongoing auditory hallucinations for 30 years, starting within a year of enduring a TBI. This patient was treated pharmacologically (i.e., with an antipsychotics and an anticonvulsant) and with group

\section{Copyright @ 2017 by Thieme Medical \\ Publishers, Inc., 333 Seventh Avenue, New York, NY 10001, USA. \\ License terms

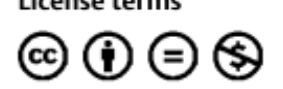

10.1055/s-0037-1606579. ISSN 2368-0539. 
psychotherapy. Improvements in mood and cognitive functioning and reduction in hallucinations were noted within 10 days of treatment, but no long-term data are available to indicate the duration of these improvements. It is also unclear if pharmacological, psychotherapeutic, or a combination of the two treatments was most effective for the patient. Antipsychotic medication was also successful in treating psychosis following a second TBI in a 51-year-old male. ${ }^{18}$ Both cases relied on second-generation antipsychotics, which have been recommended over the first-generation antipsychotics because of differences in interactions with recovering neural processes; ${ }^{19}$ the first-generation antipsychotics may decrease synaptic plasticity. ${ }^{20}$

Although there is little information on pharmacological treatment of psychosis following TBI, ${ }^{20,21}$ there is even less information on psychotherapeutic treatment. Psychotherapy as an alternative or adjunct to pharmacological treatment has shown mixed effectiveness in treating behavioral and/or mood disorders in cases of TBI (for reviews refer to ${ }^{22,23}$ ). Cognitive behavioral therapy (CBT) is one of the most commonly used psychotherapies to treat mood and behavioral symptoms in TBI cases. CBT was effective in reducing depressed and anxious mood, somatic complaints, and social problems in children and adolescents (4-18 years of age) with TBI when compared with those in individuals who did not receive psychotherapy. ${ }^{24}$ However, more rigorous studies in adult populations do not find support for CBT over other treatments. ${ }^{22,25}$

Acceptance and Commitment Therapy (ACT) may be an alternative psychotherapeutic treatment for mental health problems following TBI. ${ }^{26}$ ACT uses present moment awareness and acceptance of physical and mental symptoms to fulfill goals and live a life that the patient values. The latter piece may be a key component to recovery following TBI. ${ }^{27}$ A brief ACT intervention resulted in fewer re-hospitalizations of patients with psychosis compared with treatment as usual for up to a year, ${ }^{28,29}$ but it is unclear how effective ACT is in treating psychosis following TBI. A recent study of children with TBI and other brain injuries investigated the impact of a dual-intervention, which included family therapy and $\mathrm{ACT}^{30}$ In this study, it was found that children in the dual-intervention group had greater parent-reported improvements in both behavioral and emotional problems compared with those in a treatment as usual group. Effects were largely maintained 6 months post-baseline, but psychotic symptoms were not discussed and were likely not endorsed in these patients.

Brown et al's ${ }^{30}$ approach, using multiple systems of therapy (e.g., individual and family therapy) to address mental health problems, has also been employed as an evidence-supported treatment for those with early psychosis. ${ }^{31}$ Dynamic factors such as family functioning and social support have been found to improve outcomes for children and adults following TBI. ${ }^{32}$ Family therapy has also been claimed to be an important component in treatments of adolescents with TBI. ${ }^{33,34}$ Psychotherapeutic interventions to improve family communication ${ }^{35}$ have shown benefits in treating mood and behavioral disturbance in adolescents who have endured a $\mathrm{TBI}^{33}$ (for review refer $\mathrm{to}^{36}$ ). In addition, such interventions may help reduce distress in parents who are facing changes as their child recovers from TBI. ${ }^{37}$ However, it is unclear if multisystem psychotherapy is effective in treating TBI-related psychosis symptoms.

The following is a case of TBI-related visual and auditory hallucinations in a 14-year-old male. The patient was involved in a multisystem approach that included Vestibular-Ocular Physical Therapy, for balance and motor symptoms, as well as ACT-oriented individual and family therapies.

\section{Case Report}

\section{Patient}

A 14-year-old, Hispanic male student was referred to the Behavioral Health Clinic for the treatment of post-concussive syndrome, 4 weeks after the appearance of visual and auditory hallucinations. The adolescent presented with his third concussion, a mild TBI which he sustained during American Football practice. Prior to this, he had sustained two concussions from a basketball and a football practice within the previous 2 years. No long-term symptoms were noted following the two previous concussions. The third, most recent, concussion was the most severe for the patient. Hallucinations appeared approximately 3 weeks after the third concussion in addition to other concussive symptoms with an earlier onset (see below for more details). Parents refused pharmacological treatment for the psychotic symptoms of their son and self-referred the adolescent for psychotherapy. Written informed consent was obtained from the patient's parents prior to participation in psychotherapy. After an extensive intake interview, individual and family therapies were recommended for the patient, and his parents agreed to the treatment. The patient and parents also provided consent for presenting the case to the scientific community after the completion of the therapies.

\section{Injury}

The impact of the injury struck from below the chin, with the angle of force diagonal from the chin to top rear of the head. Magnetic resonance imaging scans without contrast, including T1-weighted and diffusion scans of the brain, were conducted 4 weeks after the injury. No abnormalities were detected. However, it is possible that this type of trauma could result in coup-contrecoup forces at the base of the brain near the basal ganglia and at the back of the brain in the parieto-occipital region, possibly causing undetected dopaminergic alteration.

\section{Symptoms}

\section{Immediate Symptoms}

The patient reported concussive symptoms in the morning following the concussion. The initial concussive symptoms were nausea, dizziness, difficulty with speech, and difficulty walking.

\section{Delayed Symptoms}

At the time of the therapy intake, 7 weeks after the injury, the patient described experiencing fatigue and difficulty with 
attention and balance. Hallucinations began 3 weeks postTBI with vivid visual and auditory components. The theme of the hallucinations was generally negative and disparaging, though not persecutory. For example, the patient often saw a horror movie-like figure who would say things such as "You will not get better." This was accompanied by the patient's own thought of "I am never going to get better." At the time of the initial individual therapy session (i.e., 7 weeks post-TBI), hallucinations appeared multiple times daily. Anxiety and depression were also present. He expressed thoughts that suggested fear of failure: "If I don't try then I will not fail." The patient expressed thoughts of self-harm. However, he denied intent to harm himself because he would not want to hurt his family emotionally. The patient took a 3-month medical leave from school $\sim 10$ weeks after the injury due to physical and cognitive symptoms and intrusive hallucinations.

\section{Assessment}

\section{Cognitive Testing}

Neuropsychological assessment was performed 11 weeks following the TBI. Testing identified strengths in shortterm and long-term memory, reasoning and problem solving, and fine motor and perceptual skills, but weaknesses in task initiation, planning and flexibility, visual processing speed, maintaining and shifting attention, and inhibition of visual and auditory distractors.

\section{Psychological Assessment}

During the intake interview, the patient reported moderate depression (Beck Depression Inventory $=27^{38}$ ) and endorsed symptoms of anxiety (i.e., sweaty palms, jitteriness, and racing heart; Beck Anxiety Inventory $=23^{39}$ ). The patient reported a pre-injury history of worry and symptoms related to anxiety that exacerbated post-injury. Some reported pre-injury worries were associated with family communications and family expectations. Therefore, family therapy was offered as another approach to managing the patient's anxiety. Family history of mental health disorders, including schizophrenia, was denied. The patient qualified for the Diagnostic and Statistical Manual of Mental Disorders, Fifth Edition (DSM-5) diagnosis 293.82 [F06.0] psychotic disorder due to TBI with hallucinations.

\section{Treatment}

\section{Vestibular-Ocular Physical Therapy}

The patient began Vestibular-Ocular Physical Therapy 1 week after the TBI to promote balance and to reduce dizziness. After completing the physical therapy course, he continued to do physical therapy exercises daily at home for 6 months (-Fig. 1).

\section{Individual Therapy}

The patient underwent 11 sessions of Acceptance and Commitment Therapy, a third-wave behavioral therapy that uses present moment awareness and acceptance of physical and mental symptoms to fulfill goals meaningful to the patient.

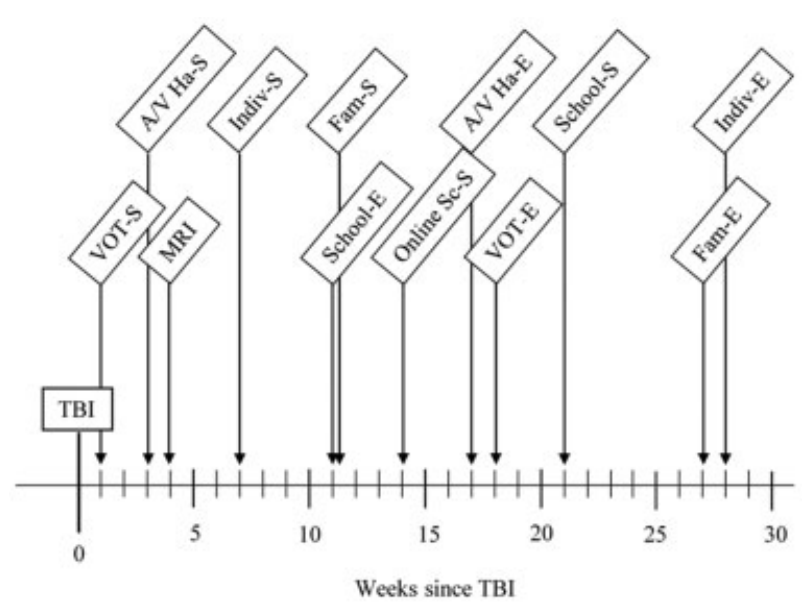

Fig. 1 Timeline of events (in weeks) after the traumatic brain injury. Abbreviations: A/V Ha, auditory/visual hallucinations; -E, end; Fam, family therapy; Indiv, individual therapy; MRI, magnetic resonance imaging; Online Sc, online schooling; -S, start; TBI, traumatic brain injury; VOT, vestibular-ocular physical therapy.

The patient was taught a problem solving approach to be used during specified 'worrying time' and was encouraged to notice hallucinations and see them simply as thoughts arising rather than symptoms of psychosis that provoke anxiety. ${ }^{28}$ A metaphor of "song stuck in the head" was used to interpret the hallucinations. The patient also learned and practiced relaxation and mindfulness exercises.

\section{Family Therapy}

The patient and his family (mother, father, and two younger siblings) completed seven sessions of family therapy. Family therapy, often added to therapies with adolescent identified patients, aimed to improve communication among all family members, de-emphasizing focus on the 'identified patient'. ${ }^{33}$ The family completed role-playing exercises in which direct and honest communication skills were practiced to reduce stress. Each family member expressed their expectations of the family unit. Then, behavioral goals were implemented to help meet each member's expectations. Compliance of behavior with goals was monitored, and barriers to achieving goals were addressed by all family members. The family also learned to notice individual bodily sensations that correspond to one's emotions. The family chose a phrase that was repeated throughout the week every time a member noticed her/himself feeling a physical sensation typically associated with anger. The goal of the exercise was to increase awareness of feelings (i.e., anger), before such feelings escalated, to make it easier to communicate with each other in a respectful and honest manner.

\section{Results}

At the beginning of the individual therapy, the therapist created 10-point 'self-rating' scales to measure post-concussive symptoms that were used throughout the treatment period ( - Table 1 ). At the end of treatment, the patient's selfrated anxiety reduced; he reported better attention, less 
Table 1 Symptom changes across a four-month period

\begin{tabular}{|l|l|l|}
\hline \multicolumn{2}{|l|}{ Initial } & Final \\
\hline Self-rating scales (up to10) & 7 & 3 \\
\hline Anxiety & 7 & 2 \\
\hline Attention difficulty & 8 & 2 \\
\hline Fatigue & 8 & 1 \\
\hline Balance difficulty & 2.5 & 1 \\
\hline Speech difficulty & 1 & 9 \\
\hline Trust in physical abilities & 8 & 7 \\
\hline Trust in mental abilities & 4 & \\
\hline Mini-MASQ & & 8 \\
\hline Distress & 14 & 10 \\
\hline Anxious arousal & 14 & 20 \\
\hline Anhedonic depression & 29 & \\
\hline
\end{tabular}

Abbreviation: Mini-MASQ, Mini-Mood and Anxiety Symptom Questionnaire.

fatigue, no difficulty with balance or speech, and higher trust in his physical and mental abilities. The patient reported reduced general distress, anxious arousal, and anhedonic depression on the Mini-Mood and Anxiety Symptom Questionnaire (Mini-MASQ ${ }^{40,41}$ ). Cognitive and psychological symptoms also improved to the point where the patient was able to return to school following a 3-month medical leave of absence. In addition, the patient's psychotic symptoms were reported to be in remission with no hallucinations experienced for more than a year (at $\sim 18$-month follow-up). Qualitative parent report suggested that the patient returned to the baseline functioning by the end of the treatment.

However, it is not clear whether the patient will experience hallucinations in the future. Psychoeducation regarding the possibility of experiencing hallucinations in the future and/or developing schizophrenia were discussed. Behavioral health resources were provided should psychotic symptoms return, and the patient and family were warned of environmental factors (e.g., drug use) that may put the patient at even greater risk for developing schizophrenia. Given that the patient had a prior history of sports-related concussion, which likely contributed to his recent TBI presentation, the topic of continuing contact sports was also discussed in individual and family therapy.

\section{Discussion}

Taken together, this case highlights the potential benefit of multisystem psychotherapeutic approaches as alternatives or complements to pharmacological treatments for post-TBI mental health complications. The patient's relatively quick remission of hallucinations, significantly decreased anxiety, and return to school feeling competent and ready after a 3-month medical leave of absence warrant further investigations into such behavioral approaches. This case is unique in several ways. The patient experienced the uncommon symptom of hallucinations following TBI, he had a previous history of head injuries, he underwent psychotherapy and did not take medications, and his psychotic symptoms were in remission after the psychotherapeutic treatment.

As in previous cases of TBI, ${ }^{11,18}$ it was important for the treatment team to distinguish early-onset schizophrenia from psychotic symptoms secondary to TBI. It is unknown whether or not the patient will experience symptoms of psychosis later in life as was the reality for the previous cases of psychosis following TBI. ${ }^{11,18}$ Given that those who have suffered a TBI are at increased risk for developing schizophrenia, ${ }^{16,17}$ it is important to provide psychoeducation to such individuals. ${ }^{42}$ Those with a history of TBI and their families should be made aware of symptoms surrounding schizophrenia-related disorders and be informed of community mental health resources for treating schizophrenia.

Should the patient's hallucinations remain in remission, differences in long-term presentation between this case and previous cases ${ }^{11,18}$ could possibly reflect the underlying brain regions affected by the TBI. Dobry et al's ${ }^{11}$ case had extensive damage to the temporal lobes, while our patient's injury showed neither magnetic resonance imaging (MRI) (at 4 weeks post-injury) nor neuropsychological assessment (at 11 weeks post-injury) evidence suggesting any temporal lobe damage/dysfunction. Fuji and Fuji's ${ }^{13}$ review of postTBI psychosis suggests that damage to the temporal lobes has the highest association with psychotic symptoms, followed by damage to the frontal lobes. Longitudinal studies are also needed to determine if multisystem psychotherapy for psychosis following a TBI would result in lower risk of developing chronic psychosis compared with those who do not participate in psychotherapy. Even if multisystem psychotherapy does not result in lower rates of chronic psychosis compared with no psychotherapy, multisystem psychotherapy may result in better ability to cope with the illness in those who partake in the therapy compared with those who do not. However, research is needed to confirm or disconfirm differences in longitudinal outcomes.

Hallucinations are difficult to treat without medications, but often medication is used as the only treatment for hallucinations. $^{31,43}$ Multisystem psychotherapeutic approaches that include medication management and psychotherapeutic approaches to address medication adherence and other behavioral issues may be most effective in treating the first-episode psychosis. ${ }^{31}$ It is not clear if the behavioral-only approaches used to treat our patient would work for other patients experiencing psychosis symptoms as a result of TBI. Several lines of research surrounding the topic are needed to better characterize treatment.

More single-case studies and randomized controlled trials are needed to determine if multisystem psychotherapy alone (i.e., without medications) would be effective in treating either psychosis following a TBI or first-episode psychosis in those who refuse pharmacological treatment.

Dismantling studies should also be pursued to identify which specific components of our multisystem treatment account for observed post-treatment changes. ${ }^{36}$ Could any psychotherapy work as effectively? Is Vestibular-Ocular Physical Therapy the key to recovery, or are all components of 
our treatment needed for the best outcomes? With constant limitations on funding for mental health care, these are questions that need answers. Psychotherapies that address family problems after TBI seem to show consistent improvements across several outcomes, ${ }^{33,44}$ and parental involvement in rehabilitation can improve outcomes. ${ }^{34}$ Involving families and/or addressing family problems as part of treatment for children and adolescents with TBI is likely beneficial because symptoms, family burden, and recovery are often interrelated for children with TBI-related symptoms. ${ }^{45}$

Our observations suggest that ACT may potentially be a viable approach to psychotherapeutic treatment for future cases of psychotic disorders following TBI, especially when combined with family therapy. ACT combined with family therapy was also found to be better than treatment as usual for reducing both behavioral and emotional problems in children with TBI. ${ }^{30}$ However, it is not clear if ACT would be better, worse, or equivalent to CBT treatment ${ }^{46,47}$ for mood and/or psychosis following TBI. In Pastore et al's ${ }^{24}$ study of CBT as treatment for behavioral and mood symptoms following $\mathrm{TBI}$, those receiving $\mathrm{CBT}$, who were not medicated, showed the greatest improvements, suggesting the possibility of interactions between psychotherapy and pharmacotherapy. Our patient did not take medications while receiving psychotherapy and had clinically significant reductions in anxiety-related symptoms. The current literature has mixed findings regarding psychotherapeutic treatments for TBI, ${ }^{22}$ which may be a product of methodological limitations, but also may be due to individual differences in treatment effectiveness as is suggested by Pastore et al. ${ }^{24}$

Investigation into individual differences in treatment response should be pursued to improve patient care and maximize resources. Several factors may contribute to individual differences, including genetic ${ }^{48}$ and psychosocial factors. ${ }^{49,50}$ Although we do not have information as to our patient's genetic susceptibility for developing psychosis, he does appear to have potential protective factors that may have contributed to his recovery. The patient's growing religious faith has been linked to better recovery compared with those without religious faith. ${ }^{51-53}$ Hispanic ethnicity is a complex and multifaceted characteristic that may or may not improve recovery post-TBI. ${ }^{54}$ He does not have a family history of psychotic disorders, which have been associated with increased risk for developing schizophrenia after a TBI. ${ }^{55}$

\section{Conclusion}

To our knowledge, this is the first non-pharmacological multisystem psychotherapeutic intervention of psychotic symptoms post mild TBI. No controlled trials have explored treatment for psychosis following TBI using longitudinal designs. Such investigations are needed to gain a better understanding of psychosis following TBI over time to develop interventions for better treating and coping with such symptoms. Dismantling studies should be conducted to identify which components of psychotherapy and Vestibular-Ocular Physical Therapy best work together and for whom. Investigations of this nature can help identify other possible contribu- tors to the patient's quick recovery (e.g., spirituality). It is important to create multi-component approaches that fit the individual patient and do not use excess resources so that we can meet the healthcare needs of more people.

\section{Conflicts of Interest}

The authors declare no conflicts of interest.

\section{Acknowledgments}

We are grateful to our patient and his family for their agreement to publication of this study.

\section{References}

1 Crowe LM, Catroppa C, Anderson V. Sequelae in children: developmental consequences. In: Grafman J, Salazar AM, eds. Handbook of Clinical Neurology: Traumatic Brain Injury Part II. Amsterdam: Elsevier BV; 2015:661-677

2 Max JE. Neuropsychiatry of pediatric traumatic brain injury. Psychiatr Clin North Am 2014;37(01):125-140

3 Crowe L, Babl F, Anderson V, Catroppa C. The epidemiology of paediatric head injuries: data from a referral centre in Victoria, Australia. J Paediatr Child Health 2009;45(06):346-350

4 Centers for Disease Control and Prevention. Nonfatal traumatic brain injuries related to sports and recreation activities among persons aged $\leq 19$ years-United States, 2001-2009. MMWR Morb Mortal Wkly Rep 2011;60(39):1337-1342

5 Crowe LM, Anderson V, Catroppa C, Babl FE. Head injuries related to sports and recreation activities in school-age children and adolescents: data from a referral centre in Victoria, Australia. Emerg Med Australas 2010;22(01):56-61

6 Polinder S, Haagsma JA, van Klaveren D, Steyerberg EW, van Beeck EF. Health-related quality of life after TBI: a systematic review of study design, instruments, measurement properties, and outcome. Popul Health Metr 2015;13(04):4

7 Prigatano GP. Psychotherapy after brain injury. In: Prigatano GP, Fordyce DJ, Zeiner HK, et al., eds. Neuropsychological Rehabilitation After Brain Injury. Baltimore, MD: Johns Hopkins University Press; 1986:67-95

8 Schachar RJ, Park LS, Dennis M. Mental health implications of traumatic brain injury (TBI) in children and youth. J Can Acad Child Adolesc Psychiatry 2015;24(02):100-108

9 Max JE, Keatley E, Wilde EA, et al. Depression in children and adolescents in the first 6 months after traumatic brain injury. Int J Dev Neurosci 2012;30(03):239-245

10 Guskiewicz KM, Broglio SP. Acute sports-related traumatic brain injury and repetitive concussion. In: Grafman J, Salazar AM, eds. Handbook of Clinical Neurology: Traumatic Brain Injury Part I. Amsterdam: Elsevier BV; 2015:157-172

11 Dobry Y, Novakovic V, Barkin RL, Sundaram VK. Management of auditory hallucinations as a sequela of traumatic brain injury: a case report and a relevant literature review. Am J Ther 2014;21 (01):e1-e6

12 Fujii D, Ahmed I. Characteristics of psychotic disorder due to traumatic brain injury: an analysis of case studies in the literature. J Neuropsychiatry Clin Neurosci 2002;14(02):130-140

13 Fujii D, Fujii DC. Psychotic disorder due to traumatic brain injury: analysis of case studies in the literature. J Neuropsychiatry Clin Neurosci 2012;24(03):278-289

14 Kim E, Lauterbach EC, Reeve A, et al; ANPA Committee on Research. Neuropsychiatric complications of traumatic brain injury: a critical review of the literature (a report by the ANPA Committee on Research). J Neuropsychiatry Clin Neurosci 2007; 19(02):106-127

15 Koufen H, Hagel KH. Systematic EEG follow-up study of traumatic psychosis. Eur Arch Psychiatry Neurol Sci 1987;237(01):2-7 
16 Molloy C, Conroy RM, Cotter DR, Cannon M. Is traumatic brain injury a risk factor for schizophrenia? A meta-analysis of casecontrolled population-based studies. Schizophr Bull 2011; 37(06):1104-1110

17 Wilcox JA, Nasrallah HA. Childhood head trauma and psychosis. Psychiatry Res 1987;21(04):303-306

18 Guerreiro DF, Navarro R, Silva M, Carvalho M, Gois C. Psychosis secondary to traumatic brain injury. Brain Inj 2009;23(04): 358-361

19 Rao V, Koliatsos V, Ahmed F, Lyketsos C, Kortte K. Neuropsychiatric disturbances associated with traumatic brain injury: a practical approach to evaluation and management. Semin Neurol 2015;35(01):64-82

20 McAllister TW, Ferrell RB. Evaluation and treatment of psychosis after traumatic brain injury. NeuroRehabilitation 2002;17(04): 357-368

21 Warden DL, Gordon B, McAllister TW, et al; Neurobehavioral Guidelines Working Group. Guidelines for the pharmacologic treatment of neurobehavioral sequelae of traumatic brain injury. J Neurotrauma 2006;23(10):1468-1501

22 Cattelani R, Zettin M, Zoccolotti P. Rehabilitation treatments for adults with behavioral and psychosocial disorders following acquired brain injury: a systematic review. Neuropsychol Rev 2010;20(01):52-85

23 Fann JR, Hart T, Schomer KG. Treatment for depression after traumatic brain injury: a systematic review. J Neurotrauma 2009; 26(12):2383-2402

24 Pastore V, Colombo K, Liscio M, et al. Efficacy of cognitive behavioural therapy for children and adolescents with traumatic brain injury. Disabil Rehabil 2011;33(08):675-683

25 Ashman T, Cantor JB, Tsaousides T, Spielman L, Gordon W. Comparison of cognitive behavioral therapy and supportive psychotherapy for the treatment of depression following traumatic brain injury: a randomized controlled trial. J Head Trauma Rehabil 2014;29(06):467-478

26 Kangas M, McDonald S. Is it time to act? The potential of acceptance and commitment therapy for psychological problems following acquired brain injury. Neuropsychol Rehabil 2011; 21(02):250-276

27 Ruff R. Selecting the appropriate psychotherapies for individuals with traumatic brain injury: what works and what does not? NeuroRehabilitation 2013;32(04):771-779

28 Bach P, Hayes SC. The use of acceptance and commitment therapy to prevent the rehospitalization of psychotic patients: a randomized controlled trial. J Consult Clin Psychol 2002;70(05):1129-1139

29 Bach P, Hayes SC, Gallop R. Long-term effects of brief acceptance and commitment therapy for psychosis. Behav Modif 2012;36(02): 165-181

30 Brown FL, Whittingham K, Boyd RN, McKinlay L, Sofronoff K. Improving child and parenting outcomes following paediatric acquired brain injury: a randomised controlled trial of Stepping Stones Triple P plus Acceptance and Commitment Therapy. J Child Psychol Psychiatry 2014;55(10):1172-1183

31 McGorry PD. Early intervention in psychosis: obvious, effective, overdue. J Nerv Ment Dis 2015;203(05):310-318

32 Holland JN, Schmidt AT. Static and dynamic factors promoting resilience following traumatic brain injury: a brief review. Neural Plast 2015;2015:902802

33 Noggle CA, Pierson EE. Psychosocial and behavioral functioning following pediatric TBI: presentation, assessment, and intervention. Appl Neuropsychol 2010;17(02):110-115

34 Braga LW, Da Paz AC, Ylvisaker M. Direct clinician-delivered versus indirect family-supported rehabilitation of children with traumatic brain injury: a randomized controlled trial. Brain Inj 2005;19(10):819-831

35 Antonini TN, Raj SP, Oberjohn KS, et al. A pilot randomized trial of an online parenting skills program for pediatric traumatic brain injury: improvements in parenting and child behavior. Behav Ther 2014;45(04):455-468

36 Brown FL, Whittingham K, Boyd R, Sofronoff K. A systematic review of parenting interventions for traumatic brain injury: child and parent outcomes. J Head Trauma Rehabil 2013;28(05):349-360

37 Brown FL, Whittingham K, Sofronoff K, Boyd RN. Parenting a child with a traumatic brain injury: experiences of parents and health professionals. Brain Inj 2013;27(13-14):1570-1582

38 Beck AT, Steer RA, Ball R, Ranieri W. Comparison of Beck Depression Inventories-IA and -II in psychiatric outpatients. J Pers Assess 1996;67(03):588-597

39 Beck AT, Epstein N, Brown G, Steer RA. An inventory for measuring clinical anxiety: psychometric properties. J Consult Clin Psychol 1988;56(06):893-897

40 Watson D, Clark LA, Weber K, Assenheimer JS, Strauss ME, McCormick RA. Testing a tripartite model: II. Exploring the symptom structure of anxiety and depression in student, adult, and patient samples. J Abnorm Psychol 1995;104(01):15-25

41 Arch JJ, Ayers CR, Baker A, Almklov E, Dean DJ, Craske MG. Randomized clinical trial of adapted mindfulness-based stress reduction versus group cognitive behavioral therapy for heterogeneous anxiety disorders. Behav Res Ther 2013;51(4-5):185-196

42 Casas RN, Gonzales E, Aldana-Aragón E, et al. Toward the early recognition of psychosis among Spanish-speaking adults on both sides of the U.S.-Mexico border. Psychol Serv 2014;11(04):460-469

43 Burns TA. What evidence is needed for service reforms in mental health care? BMJ 2005;331:586

44 Wade SL, Walz NC, Carey J, et al. Effect on behavior problems of teen online problem-solving for adolescent traumatic brain injury. Pediatrics 2011;128(04):e947-e953

45 Ganesalingam K, Yeates KO, Ginn MS, et al. Family burden and parental distress following mild traumatic brain injury in children and its relationship to post-concussive symptoms. J Pediatr Psychol 2008;33(06):621-629

46 Bacon T, Farhall J, Fossey E. The active therapeutic processes of acceptance and commitment therapy for persistent symptoms of psychosis: clients' perspectives. Behav Cogn Psychother 2014; 42(04):402-420

47 Öst LG. The efficacy of Acceptance and Commitment Therapy: an updated systematic review and meta-analysis. Behav Res Ther 2014;61:105-121

48 McAllister TW. Genetic factors in traumatic brain injury. In: Grafman J, Salazar AM, eds. Handbook of Clinical Neurology: Traumatic Brain Injury Part II. Amsterdam: Elsevier BV; 2015:723-739

49 Gordon WA, Cantor J, Dams-O'Connor K, Tsaousides T. Long-term social integration and community support. In: Grafman J, Salazar AM, eds. Handbook of Clinical Neurology: Traumatic Brain Injury Part I. Amsterdam: Elsevier BV; 2015:423-431

50 Telzer EH, Fuligni AJ, Lieberman MD, Galván A. Meaningful family relationships: neurocognitive buffers of adolescent risk taking. J Cogn Neurosci 2013;25(03):374-387

51 Galanter M, Dermatis H, Bunt G, Williams C, Trujillo M, Steinke P. Assessment of spirituality and its relevance to addiction treatment. J Subst Abuse Treat 2007;33(03):257-264

52 Lee MT, Veta PS, Johnson BR, Pagano ME. Daily spiritual experiences and adolescent treatment response. Alcohol Treat Q 2014; 32(2-3):271-298

53 Webb M, Charbonneau AM, McCann RA, Gayle KR. Struggling and enduring with God, religious support, and recovery from severe mental illness. J Clin Psychol 2011;67(12):1161-1176

54 Janus TJ, Smith HL, Chigazola A, Wortman MR, Sidwell RA, Piper JG. Hospital discharge destinations for Hispanic and non-Hispanic white patients treated for traumatic brain injury. J Trauma Nurs 2013;20(02):102-107, quiz 108-109

55 Malaspina D, Goetz RR, Friedman JH, et al. Traumatic brain injury and schizophrenia in members of schizophrenia and bipolar disorder pedigrees. Am J Psychiatry 2001;158(03):440-446 